\title{
Subset Feedback Vertex Set on Graphs of Bounded Independent Set Size
}

\author{
Charis Papadopoulos \\ Department of Mathematics, University of Ioannina, Greece \\ charis@cs.uoi.gr
}

Spyridon Tzimas

Department of Mathematics, University of Ioannina, Greece

roytzimas@hotmail.com

\begin{abstract}
The (Weighted) Subset Feedback Vertex Set problem is a generalization of the classical FEEDBACK VERTEX SET problem and asks for a vertex set of minimum (weight) size that intersects all cycles containing a vertex of a predescribed set of vertices. Although the two problems exhibit different computational complexity on split graphs, no similar characterization is known on other classes of graphs. Towards the understanding of the complexity difference between the two problems, it is natural to study the importance of a structural graph parameter. Here we consider graphs of bounded independent set number for which it is known that WEIGHTED FEEDBACK VERTEX SET can be solved in polynomial time. We provide a dichotomy result with respect to the size of a maximum independent set. In particular we show that WEIGHTED SUBSET FEEDBACK VERTEX SET can be solved in polynomial time for graphs of independent set number at most three, whereas we prove that the problem remains NP-hard for graphs of independent set number four. Moreover, we show that the (unweighted) Subset FeEdback Vertex Set problem can be solved in polynomial time on graphs of bounded independent set number by giving an algorithm with running time $n^{\mathcal{O}(d)}$, where $d$ is the size of a maximum independent set of the input graph. To complement our results, we demonstrate how our ideas can be extended to other terminal set problems on graphs of bounded independent set size. Based on our findings for Subset Feedback Vertex Set, we settle the complexity of Node Multiway Cut, a terminal set problem that asks for a vertex set of minimum size that intersects all paths connecting any two terminals, as well as its variants where nodes are weighted and/or the terminals are deletable, for every value of the given independent set number.
\end{abstract}

2012 ACM Subject Classification Mathematics of computing $\rightarrow$ Graph algorithms

Keywords and phrases Subset Feedback Vertex Set, Node Multiway Cut, Terminal Set problem, polynomial-time algorithm, NP-completeness, W[1]-hardness, graphs of bounded independent set size

Digital Object Identifier 10.4230/LIPIcs.IPEC.2018.20

Related Version A full version of the paper is available at https://arxiv.org/abs/1805. 07141, [32].

Funding This research has been financially supported by General Secretariat for Research and Technology (GSRT) and the Hellenic Foundation for Research and Innovation (HFRI) (Scholarship Code: 82220). 


\section{Introduction}

Given a (vertex-weighted) graph $G=(V, E)$ and a set $S \subseteq V$, the (Weighted) Subset FEEDBACK Vertex SET problem, introduced by Even et al. [13], asks for a vertex set of minimum (weight) size that intersects all cycles containing a vertex of $S$. Cygan et al. [10] and Kawarabayashi and Kobayashi [25] independently showed that SuBSET FEEDBACK VERTEX SET is fixed-parameter tractable (FPT) parameterized by the solution size, while Hols and Kratsch provided a randomized polynomial kernel for the problem [21]. There has been a considerable amount of work to obtain faster, still exponential-time, algorithms even when restricted to particular graph classes $[5,16,15,19]$. As a generalization of the classical FEEDBACK VERTEX SET for which $S=V$, the problem remains NP-hard on bipartite graphs [36] and planar graphs [17]. On the positive side, WeIGHTED Subset FeEdBACK VERTEX SET can be solved in polynomial time on interval graphs, permutation graphs, and cobipartite graphs [31], the latter being a subclass of graphs of independent set size at most two. However a notable difference between the two problems regarding their complexity status is the class of split graphs: FEEDBACK VERTEX SET is known to be polynomial-time solvable on split graphs [7,34], whereas Subset Feedback Vertex Set remains NP-hard on split graphs [16].

In order to obtain further (in)tractability results for Subset FEEDBACK VerTex SET, it is reasonable to consider structural parameters of graphs that may lend themselves to provide a unified approach. In terms of parameterized complexity FEEDBACK VERTEX SET is known to be FPT, when parameterized by tree-width [8] and clique-width [2] which implies that FEEDBACK VERTEX SET can be solved in polynomial time on graphs of bounded such parameters. Although FEEDBACK VERTEX SET is W[1]-hard parameterized by the size of the independent set, it can be solved in polynomial time on graphs of bounded maximum induced matching (i.e., FEEDBACK VERTEX SET belongs in XP parameterized by the size of a maximum induced matching) [24]. Only very recently, Jaffke et al. proposed an algorithm that solves Weighted FEEDBACK Vertex SET in time $n^{\mathcal{O}(w)}$ where $w$ is the maximum induced matching width of the given graph [23]. Despite their relevant name, graphs of bounded maximum induced matching (or graphs of bounded independent set number) are not related to graphs of bounded maximum induced matching width as indicated in [35].

The approach of [23] provides a powerful mechanism, as it unifies polynomial-time algorithms for WeIGHTED FEEDBACK VERTEX SET on several graph classes such as interval graphs, permutation graphs, circular-arc graphs, and Dilworth- $k$ graphs for fixed $k$, among others. Such a mechanism raises the question of whether the algorithm given in [23] can be extended to the more general setting of Weighted Subset FEedBack Vertex Set. However the proposed algorithm is based on the crucial fact that the forest of a solution has bounded number of internal nodes which is not necessarily true for the $S$-forest of WEIGHTED Subset Feedback Vertex Set. Thus it seems difficult to control the size of the solution whenever $S \subset V$. As this observation does not rule out any positive answer, here we develop the first step towards such an approach by considering graphs of bounded independent set number which form candidate relevant graphs. Although Weighted FeEdBACK Vertex SET can be solved in time $n^{\mathcal{O}(p)}$ on graphs of maximum induced matching at most $p$ [24], Subset FeEdBack VerTex SET is already NP-complete on graphs of maximum induced matching equal to one (i.e., split graphs) [16].

In this work we show that the complexity behaviour of the weighted version of the problem is completely different from the behaviour of the unweighted variant on graphs with bounded $\alpha(G)$, where $\alpha(G)$ is the size of a maximum independent set in a graph $G$. 
- We show that Weighted Subset Feedback Vertex Set can be solved in polynomial time on graphs with $\alpha(G) \leq 3$.

Such graphs consist of the complements of triangle-free graphs; recall that for triangle-free graphs Feedback Vertex Set remains NP-hard [36]. We solve Weighted Subset Feedback Vertex Set on such graphs, by exploiting a structural characterization of the solution with respect to the vertices that are close to $S$.

- We further provide a dichotomy result showing that Weighted Subset Feedback VerTeX SET remains NP-complete on graphs with $\alpha(G)=4$.

Thus we enlarge our knowledge on the complexity difference of the two problems with respect to a structural graph parameter.

- In order to complement our results we show that SubSet Feedback Vertex Set can be solved in time $n^{\mathcal{O}(d)}$, where $\alpha(G) \leq d$.

Our findings concerning Subset Feedback Vertex Set are summarized in Table 1.

Moreover, we demonstrate how our ideas can be extended to other terminal set problems on graphs of bounded independent set size. In the (unweighted) NodE Multiway Cut problem, we are given a graph $G=(V, E)$, a terminal set $T \subseteq V$, and a nonnegative integer $k$ and the goal is to find a set $X \subseteq V \backslash T$ of size at most $k$ such that any path between two different terminals intersects $X$. Node Multiway CuT is known to be in FPT parameterized by the solution size [4, 29] and even above guaranteed value [9]. For further results on variants of Node Multiway Cut we refer to [3, 18, 27]. We completely characterize the complexity of Node Multiway Cut with respect to the size of the maximum independent set.

- In particular, we show that for $\alpha(G) \leq 2$ Node Multiway Cut can be solved in polynomial time, whereas for $\alpha(G)=3$ it remains NP-complete by adopting the reduction for Weighted Subset Feedback Vertex Set with $\alpha(G)=4$.

We further consider a relaxed variation of Node Multiway Cut in which we are allowed to remove terminal vertices, called Node Multiway Cut with Deletable Terminals (also known as Unrestricted Node Multiway Cut).

- We show that the (unweighted) Node Multiway Cut with Deletable Terminals problem can be solved in polynomial time on graphs of bounded independent set number, using an idea similar to the polynomial-time algorithm for the Subset FeEdback VerTex Set problem.

- Regarding its node-weighted variation, we provide a complexity dichotomy result showing that Weighted Node Multiway Cut with Deletable Terminals can be solved in polynomial time on graphs with $\alpha(G) \leq 2$, whereas it becomes NP-complete on graphs with $\alpha(G)=3$.

We note that the polynomial-time algorithm for the weighted variation is obtained by invoking our algorithm for Weighted Subset Feedback Vertex Set on graphs with $\alpha(G) \leq 3$.

\section{Preliminaries}

We refer to $[1,11,20]$ for our standard graph terminology. For $X \subseteq V, N_{G}(X)=$ $\bigcup_{v \in X} N_{G}(v) \backslash X$ and $N_{G}[X]=N_{G}(X) \cup X$. A weighted graph $G=(V, E)$ is a graph, where each vertex $v \in V$ is assigned a weight that is a positive integer number. We denote by $w(v)$ the weight of each vertex $v \in V$. For a vertex set $A \subset V$, the weight of $A$, denoted by $w(A)$, is $\sum_{v \in A} w(v)$.

Given a graph $G$, the independent set number, denoted by $\alpha(G)$, is the size of the maximum independent set in $G$. In terms of forbidden subgraph characterization, note that $\alpha(G) \leq d$ if and only if $G$ does not contain $(d+1) K_{1}$ as an induced subgraph. We say that 
Table 1 Computational complexity results for FeEdback Vertex Set (FVS) and Subset FEEDBACK VERTEX SET (SFVS) on graphs of bounded independent set number and graphs of bounded maximum induced matching. Note that every graph of independent set number $d$ has maximum induced matching of size at most $d$, while the converse is not necessarily true.

\begin{tabular}{|c|c|c|c|}
\hline & \multicolumn{3}{|c|}{ Bounded Structural Parameter } \\
\hline & Max. Independen & Set $(d)$ & Max. Induced Matching $(p)$ \\
\hline Weighted FVS & \multicolumn{3}{|r|}{$n^{\mathcal{O}(p)}[24]$} \\
\hline \multirow{2}{*}{ Weighted SFVS } & $d \leq 3$ & Theorem 6 & \multirow[b]{3}{*}{ NP-complete $[16]$} \\
\hline & $d=4 \quad$ NP-complete & Theorem 7 & \\
\hline Unweighted SFVS & $n^{\mathcal{O}(d)}$ & Theorem 8 & \\
\hline
\end{tabular}

a graph $G$ has bounded independent set size if there exists a positive integer $d$ such that $\alpha(G) \leq d$. The clique cover number of $G$, denoted by $\kappa(G)$, is the smallest number of cliques needed to partition $V(G)$ into $S_{1}, \ldots, S_{k}$ such that $G\left[S_{i}\right]$ is a clique. A vertex cover is a set of vertices such that every edge of $G$ is incident to at least one vertex of the set. A matching is a set of edges having no common endpoint. An induced matching, denoted by $p K_{2}$, is a matching $M$ of $p$ edges such that $G[V(M)]$ is isomorphic to $p K_{2}$. The maximum induced matching number, denoted by $p(G)$, is the largest number of edges in any induced matching of $G$. It is not difficult to see that for any graph $G, \kappa(G) \geq \alpha(G) \geq p(G)$ holds.

The (Weighted) Subset Feedback Vertex Set (SFVS) problem asks for a given (vertex-weighted) graph $G=(V, E)$, a set $S \subseteq V$, and an integer $k$, whether there exists a set $X$ with $|X| \leq k(w(X) \leq k)$ such that no cycle in $G-X$ contains a vertex of $S$. As remarked, we distinguish between the weighted and the unweighted version of the problem. In the unweighted version of the problem note that all weights are equal and positive. The classical Feedback Vertex Set (FVS) problem is a special case of Subset FeEdback Vertex SET with $S=V$. A vertex of $S$ is simply called $S$-vertex. An induced cycle of $G$ is called $S$-cycle if an $S$-vertex is contained in the cycle. We define an $S$-forest $F=\left(V_{F}, E_{F}\right)$ to be the subgraph of $G$ induced by the vertex set $V_{F} \subseteq V$ for which no cycle in $G\left[V_{F}\right]$ is an $S$-cycle. It is not difficult to see that the problem of computing a minimum weighted subset feedback vertex set is equivalent to the problem of computing a maximum weighted $S$-forest.

Let us give a couple of observations on the nature of Subset Feedback Vertex Set on graphs of bounded independent set size. Let $G$ be a graph and let $d$ be a positive integer such that every independent set of $G$ has at most $d$ vertices. Firstly note that the bounded-size independent set is a hereditary property, meaning that for every induced subgraph $H$ of $G$, we have $\alpha(H) \leq d$. Moreover for any clique $C$ of $G$, any $S$-forest of $G$ contains at most two vertices of $S \cap C$.

- Observation 1. Let $G$ be a graph with $\alpha(G) \leq d$ and let $S \subseteq V$.

(1) For any set $X$ of $2 d+1$ vertices, there is a cycle in $G[X]$.

(2) Any $S$-forest of $G$ has at most $2 d$ vertices from $S$.

We note that Observation 1 directly implies that any $2 d+1$ vertices of $G[S]$ induce an $S$-cycle, which allows us to construct by brute force all possible subsets of $S$ belonging to any $S$-forest in time $n^{\mathcal{O}(d)}$. 


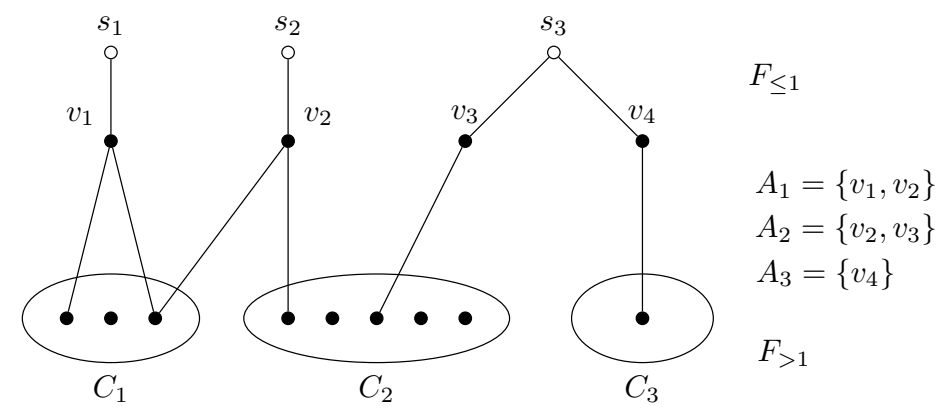

Figure 1 Illustrating an $S$-distance partition $\left(F_{\leq 1}, F_{>1}\right)$ of an $S$-forest $F$ with $S=\left\{s_{1}, s_{2}, s_{3}\right\}$ that shows the connected components $C_{1}, C_{2}, C_{3}$ of $F_{>1}$. The edges inside $F_{>1}$ are not drawn in order to highlight that the cut satisfies the given tuple $\left(A_{1}, A_{2}, A_{3}\right)$.

\section{Weighted SFVS on Graphs of Bounded Independent Set}

Here we consider the Weighted Subset Feedback Vertex Set and we show a dichotomy result with respect to the size of the maximum independent set. We first provide a polynomialtime algorithm on graphs of independent set size at most three and then we show that Weighted Subset Feedback Vertex Set is NP-complete on graphs of independent set size equal to four.

Let $(G, S, k)$ be an instance of Weighted Subset Feedback Vertex Set for which $G$ is a graph of independent set size at most $d$. In the forthcoming arguments, instead of directly computing a solution for Weighted Subset Feedback Vertex Set, we consider the equivalent problem of computing an $S$-forest of $G$ having weight at least $w(V)-k$.

Let $F=\left(V_{F}, E_{F}\right)$ be an induced subgraph of $G$. Recall that an $S$-forest is an induced subgraph of $G$. We partition the graph $F$ into two induced subgraphs $F_{\leq 1}$ and $F_{>1}$ as follows:

- $F_{\leq 1}$ is the subgraph of $F$ induced by the vertices of $N\left[S \cap V_{F}\right]$; the vertices of $F_{\leq 1}$ are at distance at most one from $S \cap V_{F}$ and are denoted by $S_{\leq 1}$.

- $F_{>1}$ is the graph $F-S_{\leq 1}$ and contains vertices that are at distance at least two from $S \cap V_{F}$.

Such a partition is called the $S$-distance partition of $F$, denoted by $\left(F_{\leq 1}, F_{>1}\right)$. The set of edges of $F$ having one endpoint in $F_{\leq 1}$ and the other in $F_{>1}$ are called the cut with respect to $F_{\leq 1}$ and $F_{>1}$. Notice that a vertex of $F_{\leq 1}$ that is adjacent to a vertex of $F_{>1}$ belongs to $S \leq 1 \backslash S$.

Let $\left\{C_{1}, \ldots, C_{d^{\prime}}\right\}$ be the partition of the vertices of $F_{>1}$ such that each $C_{i}, 1 \leq i \leq d^{\prime}$, induces a connected component in $F_{>1}$. Because $F_{>1}$ is an induced subgraph of $G$, it is clear that $d^{\prime} \leq d$. Let $\left(A_{1}, \ldots, A_{d^{\prime}}\right)$ be a tuple of $d^{\prime}$ subsets of $S_{\leq 1} \backslash S$, i.e., each $A_{i} \subseteq\left(S_{\leq 1} \backslash S\right)$ holds. We say that the cut satisfies the tuple $\left(A_{1}, \ldots, A_{d^{\prime}}\right)$ if for any vertex $v \in C_{i}$, we have $\left(N_{G}(v) \cap S_{\leq 1}\right) \subseteq A_{i}$. The notion of an $S$-distance partition of $F$ with the corresponding cut is illustrated in Figure 1.

We now utilize the $S$-distance partition of $F$ in order to construct an algorithm that solves Weighted Subset Feedback Vertex Set on graphs of independent set size at most $d$ and subsequently show that this algorithm is efficient for $d \leq 3$. Our general approach relies on the following facts:

- By Observation 1 (2) we try all subsets $S^{\prime}$ of $S$ with at most $2 d$ vertices and keep those sets that induce a forest. This step is used in constructing the graph $F_{\leq 1}$. In particular, 
for each such set $S^{\prime}$, we construct all $F_{\leq 1}$ such that $S \cap V\left(F_{\leq 1}\right)=S^{\prime}$. We will show that the number of such subsets produced is bounded by $n^{\mathcal{O}(d)}$.

- For each of the potential subsets $S^{\prime}$ constructed in the previous step, and for each $d^{\prime} \leq d$, we determine all possible tuples $\left(A_{1}, \ldots, A_{d^{\prime}}\right)$ in $F_{\leq 1}$ with $A_{i} \subseteq\left(N\left[S^{\prime}\right] \backslash S\right)$ that are satisfied by cuts of $S$-distance partitions. We show why considering only these tuples is sufficient in Lemma 2.

- Up to that point we can show that all steps can be executed in polynomial time regardless of $d \leq 3$. However for the next and final step we can only achieve polynomial running time if we restrict ourselves to $d \leq 3$ due to the number of connected components of $F_{>1}$. For each tuple computed in the previous step, we find connected components $C_{1}, \ldots, C_{d^{\prime}}$ of maximum weight such that the cut of $\left(G\left[S^{\prime}\right], G\left[C_{1} \cup \cdots \cup C_{d^{\prime}}\right]\right)$ satisfies the tuple. For doing so, we take advantage of the small number of connected components $\left(d^{\prime}<3\right)$ and an efficient way of computing a vertex-cut between such components.

We begin by showing that the $S$-distance partition of $F$ provides a useful tool towards computing a maximum $S$-forest. Given a set of vertices $X \subseteq N[S]$ and $d^{\prime}$ subsets $A_{i}$ of $X \backslash S$, we construct the graph $\widehat{G}$ that is obtained from $G[X]$ by adding $d^{\prime}$ vertices $w_{1}, \ldots, w_{d^{\prime}}$ such that every vertex $w_{i}$ is adjacent to all the vertices of $A_{i}$. In what follows, we always assume that $G$ is a graph having independent set size at most $d$.

- Lemma 2. Let $F$ be an $S$-forest of $G$ with $S$-distance partition $\left(F_{\leq 1}, F_{>1}\right)$ such that $S_{\leq 1} \cap S \neq \varnothing$. Then for $d^{\prime} \leq d$, there is a tuple $\left(A_{1}, \ldots, A_{d^{\prime}}\right)$ with $A_{i} \subseteq\left(S_{\leq 1} \backslash S\right)$ such that (i) the cut of $\left(F_{\leq 1}, F_{>1}\right)$ satisfies $\left(A_{1}, \ldots, A_{d^{\prime}}\right)$ and

(ii) every induced subgraph $H$ of $G$ with $S$-distance partition $\left(H\left[S_{\leq 1}\right], H-S_{\leq 1}\right)$ that satisfies $\left(A_{1}, \ldots, A_{d^{\prime}}\right)$ is an $S$-forest.

Proof. Let $\left\{C_{1}, \ldots, C_{d^{\prime}}\right\}$ be the partition of the vertices of $F_{>1}$ such that every $C_{i}$ induces a connected component in $F_{>1}$. We define a tuple $\left(A_{1}, \ldots, A_{d^{\prime}}\right)$ in which every $A_{i}=$ $N\left(C_{i}\right) \cap S_{\leq 1}$, for $1 \leq i \leq d^{\prime}$. Clearly $A_{i} \subseteq\left(S_{\leq 1} \backslash S\right)$ since every vertex $F_{>1}$ is at distance at least two from $S_{\leq 1} \cap S$. Thus, by construction, the cut of $\left(F_{\leq 1}, F_{>1}\right)$ satisfies the tuple $\left(A_{1}, \ldots, A_{d^{\prime}}\right)$.

For the next claim, we first show that $\widehat{G}$ with respect to $S_{\leq 1}$ and the tuple $\left(A_{1}, \ldots, A_{d^{\prime}}\right)$ is an $S$-forest. Assume for contradiction that there is an $S$-cycle $\widehat{C}$ in $\widehat{G}$. Since $F_{\leq 1}$ does not contain any $S$-cycle, $\widehat{C}$ contains a vertex $w_{i}$ and at least two vertices $u_{i}, v_{i}$ from $A_{i}$, $1 \leq i \leq d^{\prime}$. By the fact that $A_{i}=N\left(C_{i}\right) \cap S_{\leq 1}$, there is a vertex $x$ in $C_{i}$ of $F_{>1}$ that is adjacent to $u_{i}$ and there is a vertex $y$ in $C_{i}$ of $F_{>1}$ that is adjacent to $v_{i}$. Together with a path between $x$ and $y$ in the connected component $C_{i}$, we construct a path in $G$ with endvertices $u_{i}$ and $v_{i}$ that is completely contained in $C_{i}$. This means that if we replace every vertex $w_{i}$ of $\widehat{C}$ by a path with internal vertices of $C_{i}$ then we obtain an $S$-cycle in $F$, leading to a contradiction. Thus, $\widehat{G}$ is an $S$-forest.

Let $H$ be an induced subgraph of $G$ with $S$-distance partition $\left(H\left[S_{\leq 1}\right], H-S_{\leq 1}\right)$ that satisfies $\left(A_{1}, \ldots, A_{d^{\prime}}\right)$. Observe that $H\left[S_{\leq 1}\right]=F_{\leq 1}$ as they are induced subgraphs of the same vertex set of $G$. Thus $H\left[S_{\leq 1}\right]$ does not contain any $S$-cycle, because $F$ is an $S$-forest. Since the cut of $\left(H\left[S_{\leq 1}\right], H-S_{\leq 1}\right)$ satisfies $\left(A_{1}, \ldots, A_{d^{\prime}}\right)$, there is a partition $\left\{T_{1}, \ldots, T_{d^{\prime}}\right\}$ in $H-S_{\leq 1}$ such that $T_{i}$ is a connected component of $H-S_{\leq 1}$ and $N\left(T_{i}\right) \subseteq A_{i}$, for $1 \leq i \leq d^{\prime}$. We show that $H$ is indeed an $S$-forest. For contradiction, assume an $S$-cycle $C$ in $H$. There are no $S$-cycles in $H\left[S_{\leq 1}\right]$ which implies that $C \cap T_{i} \neq \varnothing$ for some $1 \leq i \leq d^{\prime}$. For every such set we replace the part $C \cap T_{i}$ by a vertex $w_{i}^{\prime}$. Denote by $H^{\prime}$ the resulting graph. Notice that $H^{\prime}[C]$ is a subgraph of $\widehat{G}[C]$ because $N_{H^{\prime}}\left(w_{i}^{\prime}\right) \subseteq N_{\widehat{G}}\left(w_{i}\right)$. This, however, implies an $S$-cycle in $\widehat{G}$ which gives the desired contradiction. Therefore, $H$ is an $S$-forest. 
Notice that $G-S$ is trivially an $S$-forest of $G$. Thus, if $F$ is an $S$-forest of $G$ such that $S_{\leq 1} \cap S=\varnothing$ then $F$ does not contain any vertex of $S$ and $F=G-S$. Next, we assume that $S_{\leq 1} \cap S \neq \varnothing$ and show how to bound the vertex set $S_{\leq 1}$ of $F_{\leq 1}$.

- Lemma 3. Let $F$ be an $S$-forest of $G$ with $S$-distance partition $\left(F_{\leq 1}, F_{>1}\right)$ such that $S_{\leq 1} \cap S \neq \varnothing$.

1. If $\left|S_{\leq 1} \cap S\right| \leq 2 d-2$ then $\left|S_{\leq 1}\right| \leq 4 d-2$.

2. If $\left|S_{\leq 1} \cap S\right| \geq 2 d-1$ then $\left|S_{\leq 1}\right| \leq 2 d$.

Proof. Let $F$ be such an $S$-forest of $G$ with $\left|S_{\leq 1} \cap S\right| \geq 1$. By Observation 1 (2), we know that $\left|S_{\leq 1} \cap S\right| \leq 2 d$. To ease the presentation, we let $S^{\prime}=S_{\leq 1} \backslash S$. We consider separately the two cases of the claim.

Case 1. Let $1 \leq\left|S_{\leq 1} \cap S\right| \leq 2 d-2$. Assume for contradiction that $\left|S^{\prime}\right|>4 d-\left|S_{\leq 1} \cap S\right|-2$. We show that $F\left[S^{\prime}\right]$ contains a matching with at least $d$ edges. Observe that $\left|S^{\prime}\right|+\left|S_{\leq 1} \cap S\right|>$ $4 d-2$. Applying Observation 1 (1) shows that there is a cycle $C$ in $F\left[S_{\leq 1}\right]$. Since $F$ is an $S$-forest, this is not an $S$-cycle, so all vertices contained in $C$ are vertices of $S^{\prime}$. Iteratively removing the two endpoints of an edge from $C$, as long as $\left|S^{\prime}\right|+\left|S_{\leq 1} \cap S\right|>2 d$, constructs $d$ edges of $S^{\prime}$ having no common endpoints by Observation 1 (1). Thus, $F\left[S^{\prime}\right]$ contains a matching $M$ with at least $d$ edges.

Let $C_{1}, \ldots, C_{d^{\prime}}$ be the connected components of $F\left[S_{\leq 1} \cap S\right]$. Notice that $d^{\prime} \leq d$ because $F\left[S_{\leq 1} \cap S\right]$ is an induced subgraph of a graph with maximum independent set size at most $d$. By construction, every vertex of $S^{\prime}$ is adjacent to at least one vertex of $S_{\leq 1} \cap S$. If the endpoints of an edge of $M$ in $S^{\prime}$ are adjacent to vertices of the same component $C_{i}, 1 \leq i \leq d^{\prime}$, then there is an $S$-cycle in $F$ since every vertex of $C_{i}$ belongs to $S$. Thus the endpoints of every edge of $M$ are adjacent to different connected components of $F\left[S_{\leq 1} \cap S\right]$. Now obtain a bipartite graph by contracting every component $C_{i}$ into a single vertex and every edge of $M$ into a single vertex and keep only the adjacencies between the components and the edges of $M$. Let $(A, B)$ be the bipartition of the resulting bipartite graph such that $A$ contains the components of $F\left[S_{\leq 1} \cap S\right]$ and $B$ contains the edges of $M$. Since $|A| \leq|B|$ and every vertex of $B$ is adjacent to at least two vertices of $A$, there is a cycle in the bipartite graph. Then, it is not difficult to see that the cycle of the contracted vertices corresponds to an $S$-cycle in $F$. Therefore there is an $S$-cycle in an $S$-forest, leading to a contradiction.

Case 2. Let $2 d-1 \leq\left|S_{\leq 1} \cap S\right| \leq 2 d$. Assume for contradiction that $\left|S^{\prime}\right|>2 d-\left|S_{\leq 1} \cap S\right|$. This means that $S^{\prime}$ contains at least one vertex. We pick a nonempty subset $W$ of $S^{\prime}$ as follows. If $\left|S_{\leq 1} \cap S\right|=2 d-1$ then $W$ consists of any two vertices of $S^{\prime}$. If $\left|S_{\leq 1} \cap S\right|=2 d$ then $W$ consists of an arbitrary vertex of $S^{\prime}$. In both cases, notice that $\left|S_{\leq 1} \cap S\right|+|W|>2 d$ by the fact $2 d-1 \leq\left|S_{\leq 1} \cap S\right|$. Then Observation 1 (1) implies that there is a cycle in $F\left[\left(S_{\leq 1} \cap S\right) \cup W\right]$. Since $W$ has at most two vertices, we conclude that the induced cycle of $F\left[\left(S_{\leq 1} \cap S\right) \cup W\right]$ has at least one vertex from $S$, hence it is an $S$-cycle in $F$. Therefore we reach a contradiction which implies that $\left|S^{\prime}\right| \leq 2 d-\left|S_{\leq 1} \cap S\right|$.

Lemma 3 shows that we can compute all possible candidates for $S_{\leq 1}$ in polynomial time as follows.

- We first try, by brute force, all subsets $S^{\prime}$ of $S$ having at most $2 d$ vertices, according to Observation 1 (2).

- Then, for each such subset $S^{\prime}$, we incorporate a set $X^{\prime} \subseteq N\left(S^{\prime}\right)$ for which either $\left|X^{\prime}\right|+\left|S^{\prime}\right| \leq 4 d-2$, or $\left|X^{\prime}\right|+\left|S^{\prime}\right| \leq 2 d$, according to Lemma 3 .

- Given the described sets $S^{\prime}$ and $X^{\prime}$, we check if $G\left[S^{\prime} \cup X^{\prime}\right]$ induces an $S$-forest and, if so, we include them into a list $L_{1}$ containing all candidates for $S_{\leq 1}$. 
The correctness follows from Observation 1 and Lemma 3. Regarding the running time, notice that we create at most $n^{\mathcal{O}(d)}$ subsets for each of $S^{\prime}$ and $X^{\prime} \subseteq N\left(S^{\prime}\right)$. Thus, in time $n^{\mathcal{O}(d)}$ we can compute a list $L_{1}$ that contains all possible subsets of the vertices corresponding to $S_{\leq 1}$. Notice that such vertices are enough to build the part $F_{\leq 1}$.

Let $S_{\leq 1}$ be a set of $L_{1}$. We now focus on the graph $G^{\prime}=G-\left(S_{\leq 1} \cup S\right)$ that contains the vertices that are at distance of at least two from $S_{\leq 1} \cap S$. Observe that for any $S$-forest $F$, the set of all vertices in $F$ which are at distance of at most one from the vertices of $S \cap V(F)$, are present as an element of $L_{1}$. Let $d^{\prime}$ be the number of connected components of $G^{\prime}$. It is clear that $d^{\prime} \leq d$. In fact, if $S_{\leq 1} \cap S$ contains at least one vertex then $d^{\prime}<d$, since the vertices of $G^{\prime}$ are at distance of at least two from $S_{\leq 1} \cap S$. Moreover, observe that if $S_{\leq 1} \cap S=\varnothing$ then $G-S$ is a trivial solution, since we try all subsets of $S$, having at most $2 d$ vertices, for the set $S_{\leq 1}$. From now on, we assume that $\left|S_{\leq 1} \cap S\right| \geq 1$ so that $d^{\prime}<d$.

By brute force, we find all tuples $\left(A_{1}, \ldots, A_{d^{\prime}}\right)$ such that the following hold:

(i) $A_{i} \subseteq\left(S_{\leq 1} \backslash S\right)$, for every $1 \leq i \leq d^{\prime}$, and

(ii) the graph $\widehat{G}$ with respect to $S_{\leq 1}$ and $\left(A_{1}, \ldots, A_{d^{\prime}}\right)$ is an $S$-forest.

Notice that by Lemma 2 (ii) it is sufficient to consider only such tuples. Since $A_{i} \subseteq S_{\leq 1}$, $d^{\prime}<d$, and $\left|S_{\leq 1}\right| \leq 4 d$, the number of tuples is $d^{\mathcal{O}(d)}$, so that we can obtain the desired set of tuples that satisfy both conditions in polynomial time.

In what follows, we consider the case for $d \leq 3$. By the previous arguments, we are given a set $S_{\leq 1} \subseteq N[S]$ and tuples of the form $A_{1}$ or $\left(A_{1}, A_{2}\right)$ which are subsets of $S_{\leq 1} \backslash S$. Our task is to compute a subset $V^{\prime}$ of the vertices of $G^{\prime}$ such that the vertices of $S_{\leq 1} \cup V^{\prime}$ induce a maximum $S$-forest and the cut $\left(G\left[S_{\leq 1}\right], G\left[V^{\prime}\right]\right)$ satisfies $A_{1}$ or $\left(A_{1}, A_{2}\right)$, respectively. We distinguish the two cases with the following two lemmas.

- Lemma 4. Let $X \subseteq N[S]$ and let $A_{1}$ be a subset of $X \backslash S$ such that both $F_{\leq 1}=G[X]$ and $\widehat{G}$ with respect to $X$ and $A_{1}$ are $S$-forests. There exists a polynomial-time algorithm that computes a maximum $S$-forest $F$ with an $S$-distance partition $\left(F_{\leq 1}, F_{>1}\right)$ having a cut satisfying $A_{1}$.

Proof. Since $F_{\leq 1}$ is a fixed $S$-forest of $F$, we need to determine the vertices of $V \backslash(X \cup S)$ that are included in $F_{>1}$. By the desired cut of $\left(F_{\leq 1}, F_{>1}\right)$, we are restricted to the vertices of $V \backslash(X \cup S)$ that have neighbors in $F_{\leq 1}$ only to $A_{1}$. Those vertices can be described as follows:

$$
B_{1}=(V \backslash(X \cup S)) \backslash\left\{w \in V: N(w) \cap\left(X \backslash\left(S \cup A_{1}\right)\right) \neq \varnothing\right\} .
$$

Notice that $B_{1}$ contains vertices that are at distance at least two from the $S$-vertices of $X \cap S$. Since the cut satisfies a single subset $A_{1}$, we have at most one connected component of $G\left[B_{1}\right]$ in $F_{>1}$. In order to choose the correct connected component of $G\left[B_{1}\right]$, we try to include each of them in $F_{>1}$ and select the one having the maximum total weight. Notice that adding any component of $G\left[B_{1}\right]$ into $F_{>1}$ cannot create any $S$-cycle, because $\widehat{G}$ with respect to $X$ and $A_{1}$ is an $S$-forest. Thus, by Lemma 2, we correctly compute a maximum $S$-forest with the desired properties. Clearly the set $B_{1}$ can be constructed in polynomial time. Since the number of connected components $G\left[B_{1}\right]$ is at most two, all steps can be executed in polynomial time.

- Lemma 5. Let $X \subseteq N[S]$ and let $A_{1}, A_{2}$ be subsets of $X \backslash S$ such that both $F_{\leq 1}=G[X]$ and $\widehat{G}$ with respect to $X$ and $\left(A_{1}, A_{2}\right)$ are $S$-forests. There exists a polynomial-time algorithm that computes a maximum $S$-forest $F$ with an $S$-distance partition $\left(F_{\leq 1}, F_{>1}\right)$ having a cut satisfying $\left(A_{1}, A_{2}\right)$. 
Proof. Similar to the proof of Lemma 4 , we first construct the sets $B_{1}, B_{2}$ that contain vertices of $V \backslash(X \cup S)$ and satisfy the cut obtained from $X$ :

$$
\begin{aligned}
& B_{1}=(V \backslash(X \cup S)) \backslash\left\{w \in V: N(w) \cap\left(X \backslash\left(S \cup A_{1}\right)\right) \neq \varnothing\right\} \text { and } \\
& B_{2}=(V \backslash(X \cup S)) \backslash\left\{w \in V: N(w) \cap\left(X \backslash\left(S \cup A_{2}\right)\right) \neq \varnothing\right\} .
\end{aligned}
$$

As the desired cut of $\left(F_{\leq 1}, F_{>1}\right)$ satisfies $\left(A_{1}, A_{2}\right)$, there are two connected components of $F_{>1}$ which are subsets of the two sets $B_{1}$ and $B_{2}$, respectively. Let $C_{1}$ and $C_{2}$ be the connected components of $F_{>1}$ such that $C_{1} \subseteq B_{1}$ and $C_{2} \subseteq B_{2}$. Now observe that there should be two non-adjacent vertices $w_{1} \in B_{1}$ and $w_{2} \in B_{2}$ that belong to $C_{1}$ and $C_{2}$, respectively. We iterate over all possible pairs of non-adjacent vertices $w_{1} \in B_{1} \cap C_{1}$ and $w_{2} \in B_{2} \cap C_{2}$ in $\mathcal{O}\left(n^{2}\right)$ time. Assuming a given choice for $w_{1}$ and $w_{2}$, observe the following:

- Since $w_{1}$ and $w_{2}$ are vertices of different connected components of $F_{>1}$, the components themselves are further restricted to be subsets of $B_{1} \backslash N\left[w_{2}\right]$ and $B_{2} \backslash N\left[w_{1}\right]$, respectively. That is, $C_{1} \subseteq\left(B_{1} \backslash N\left[w_{2}\right]\right)$ and $C_{2} \subseteq B_{2} \backslash N\left[w_{1}\right]$.

- Since $F$ has at least one vertex of $S, w_{1}, w_{2} \in V \backslash(X \cup S)$ are non-adjacent, and by the fact $d \leq 3$, we have that $B_{1} \backslash N\left[w_{2}\right]$ and $B_{2} \backslash N\left[w_{1}\right]$ induce cliques in $G$. Thus $B_{1} \backslash N\left[w_{2}\right] \subseteq N\left[w_{1}\right]$ and $B_{2} \backslash N\left[w_{1}\right] \subseteq N\left[w_{2}\right]$, respectively.

Then by the second statement it is not difficult to see that $B_{1} \backslash N\left[w_{2}\right]$ and $B_{2} \backslash N\left[w_{1}\right]$ are disjoint. Let $B_{1}^{\prime}=\left(B_{1} \backslash N\left[w_{2}\right]\right) \backslash\left\{w_{1}\right\}$ and $B_{2}^{\prime}=\left(B_{2} \backslash N\left[w_{1}\right]\right) \backslash\left\{w_{2}\right\}$. Now in order to find the maximum induced $S$-forest under the stated conditions and our assumption that $w_{1}$ and $w_{2}$ belong to the two connected components of $F_{>1}$, it suffices to find the maximum subset $C_{1} \cup C_{2}$ of $B_{1}^{\prime} \cup B_{2}^{\prime}$ such that there are no edges between the vertices of $C_{1} \cap B_{1}^{\prime}$ and the vertices of $C_{2} \cap B_{2}^{\prime}$. This boils down to compute a minimum weighted vertex cover on the bipartite graph $G^{\prime}$ obtained from $G\left[B_{1}^{\prime} \cup B_{2}^{\prime}\right]$ and removing the edges inside $G\left[B_{1}^{\prime}\right]$ and $G\left[B_{2}^{\prime}\right]$. By maximum flow standard techniques, we compute a minimum weighted vertex cover $U$ on $G^{\prime}$ in polynomial time [30]. Therefore, $G\left[B_{1}^{\prime} \cup B_{2}^{\prime}\right]-U$ contains the connected components $C_{1} \backslash\left\{w_{1}\right\}$ and $C_{1} \backslash\left\{w_{2}\right\}$, as required.

Now we are equipped with our necessary tools in order to obtain our main result, namely a polynomial-time algorithm that solves Weighted Subset FEedBACK VERTEx SET on graphs of independent set of size at most 3 .

Theorem 6. Weighted Subset Feedback Vertex SeT on graphs of independent set of size at most 3 can be solved on time $n^{\mathcal{O}(1)}$.

Proof. Let us briefly explain such an algorithm for computing a maximum $S$-forest $F$ of a graph $G$ having independent set size at most three. Let $d=3$. Initially we set $F^{*}=G-S$. Then, for every set $X \subseteq N[S]$ with $|X| \leq 4 d$ such that $G[X]$ is an $S$-forest, we try by brute force all subsets $A_{1}$ and $\left(A_{1}, A_{2}\right)$ with $A_{i} \subseteq(X \backslash S)$ such that $\widehat{G}$ with respect to $X$ and $A_{1}$ or $\left(A_{1}, A_{2}\right)$ is an $S$-forest. For each of such subsets, we find a maximum $S$-forest $F$ with an $S$-distance partition $\left(G[X], F_{>1}\right)$ having a cut satisfying $A_{1}$ or $\left(A_{1}, A_{2}\right)$, respectively, by applying the algorithms described in Lemma 4 and Lemma 5 . At each step, we maintain the maximum weighted $S$-forest $F^{*}$ by comparing $F$ with $F^{*}$. Finally we provide the vertices $V \backslash V\left(F^{*}\right)$ as the set with the minimum total weight that are removed from $G$.

By Lemma 3, it is sufficient to consider the described subsets $X$. Since every induced subgraph of $G-X$ contains at most two connected components, Lemma 2 implies that all possible subsets $A_{1}$ or $\left(A_{1}, A_{2}\right)$ with the described properties are enough to consider. Thus, the correctness follows from Lemmata $3-5$. Regarding the running time, notice that whether a graph contains an $S$-cycle can be tested in linear time. Thus we can construct all 
described and valid subsets in $n^{\mathcal{O}(1)}$ time. Therefore the total running time of the algorithm is $n^{\mathcal{O}(1)}$, since each of the algorithms given in Lemma 4 and Lemma 5 , respectively, requires polynomial time.

Let us now show that extending Theorem 6 to graphs of larger independent sets is not possible. More precisely, with the following result we show that Weighted Subset Feedback Vertex Set is para-NP-complete parameterized by $\alpha(G)$.

- Theorem 7. Weighted Subset Feedback Vertex Set is NP-complete on graphs of independent set of size at most 4.

Proof. We will provide a polynomial reduction from the VerTex Cover problem on tripartite graphs which is NP-complete [17] and asks whether a tripartite graph $G$ contains a vertex cover of weight at most $k$. Let $G=(A, B, C, E)$ be a tripartite graph on $n$ vertices, where $(A, B, C)$ is the partition of $V(G)$. We construct a weighted graph $G^{\prime}$ from $G$ in polynomial time as follows.

- We turn the three independent sets $A, B$ and $C$ into cliques by adding all necessary edges and we give all vertices unary weight.

- We add a vertex $r_{A}$ that is adjacent to all of the vertices of $A$ and we assign weight $n$ to $r_{A}$. In a completely symmetric way, we add vertices $r_{B}$ and $r_{C}$ with respect to the sets $B$ and $C$, respectively.

- We add a vertex $s$ that is adjacent to all three vertices $r_{A}, r_{B}, r_{C}$ having weight $n$.

This completes the construction of $G^{\prime}$. Observe that all vertices of $V\left(G^{\prime}\right) \backslash\left\{s, r_{A}, r_{B}, r_{C}\right\}$ have weight equal to one. It is not difficult to verify that the constructed graph $G^{\prime}$ is a graph having an independent set at most 4 , since the vertex set of $G^{\prime}-\{s\}$ can be partitioned into three cliques.

Next we claim that $G$ has a vertex cover $U$ of weight at most $k<n$ if and only if $G^{\prime}$ with $S=\{s\}$ has a subset feedback vertex set of weight at most $k$. Assume a vertex cover $U$ of $G$. By definition, $U$ covers all edges of $G$, so that $G[(A \cup B \cup C) \backslash U]$ is an independent set. This means that $G^{\prime}[(A \cup B \cup C) \backslash U]$ is a vertex-disjoint union of cliques. Since $s$ is non-adjacent to any vertex of $G$ and $G^{\prime}\left[r_{A}, r_{B}, r_{C}\right]$ is an independent set, every cycle of $G^{\prime}-U$ contains a vertex of $r_{A}, r_{B}$ and $r_{C}$ with at least two vertices from $A, B$ and $C$, respectively. Thus, $G^{\prime}-U$ is a connected $S$-forest. Therefore, $U$ is a subset feedback vertex set of $\left(G^{\prime},\{s\}\right)$ of size at most $k$.

For the opposite direction, assume a subset feedback vertex set $F$ of $\left(G^{\prime},\{s\}\right)$. If $F$ is not a subset of $A \cup B \cup C$, then its sum of weights is greater or equal to $n$. Then $F$ is not a minimum subset feedback vertex set of $\left(G^{\prime},\{s\}\right)$, since $A \cup B \cup C$ minus a single vertex is trivially a subset feedback vertex set of $\left(G^{\prime},\{s\}\right)$ of total weight $n-1$. Thus $F$ is indeed a subset of $A \cup B \cup C$. Assume that $F$ is not a vertex cover of $G$. By definition, there is an edge of $G$ that remains uncovered. Without loss of generality, assume that this edge has its endpoints on the vertices $x \in A$ and $y \in B$. Then $\left\langle s, r_{A}, x, y, r_{B}\right\rangle$ is an induced cycle of $G^{\prime}$, which contradicts the fact that $F$ is a subset feedback vertex set of $\left(G^{\prime},\{s\}\right)$. Therefore $F$ is a vertex cover of $G$.

We stress that Theorem 7 further implies that the NP-completeness result carries along to graphs of clique cover number at most four, since the constructed graph given in the proof can be partitioned into four disjoint cliques. 


\section{SFVS on Graphs of Bounded Independent Set}

Here we show that despite the complexity dichotomy result for the Weighted Subset FEEDBACK VERTEX SET, whenever the weights of the vertices are equal Subset FEEDBACK VERTEX SET can be solved in polynomial time on graphs of bounded independent set number.

- Theorem 8. Subset FEedBACK VERTEx SET on graphs of independent set of size at most $d$ can be solved in time $n^{\mathcal{O}(d)}$.

Proof. Let $G=(V, E)$ be a graph with $\alpha(G) \leq d$ and let $S \subseteq V$. Denote by $X \subseteq V$ a minimum subset feedback vertex set of $G$. Let $F=G-X$ be a maximum $S$-forest of $G$. By Observation 1 (2), the vertices of $S$ that belong to $F$ are at most $2 d$. Thus for every optimum solution $X$, the set $S \backslash X$ has at most $2 d$ vertices.

Now we claim that it is enough to try guessing all subsets $X^{\prime}$ of $X$ for which $\left|X^{\prime}\right| \leq 2 d$. To see this, observe that if $X \backslash S$ has order more than $2 d$, then $G-S$ has more vertices than $G-X$, leading to a contradiction to the optimality of $X$. Hence, $X \backslash S$ has at most $2 d$ vertices. In order to find an optimal solution, it suffices to consider all such candidates $S^{\prime}$ for $S \backslash X$ and $X^{\prime}$ for $X \backslash S$. To check whether an induced subgraph of $G$ consists an $S$-forest takes $\mathcal{O}(n+m)$ time. Since the number of such sets $S^{\prime}$ is at most $n^{2 d}$ and the number of the considered sets $X^{\prime}$ is at most $n^{2 d}$, the total running time is bounded by $n^{\mathcal{O}(d)}$. Therefore in time $n^{\mathcal{O}(d)}$ we compute a minimum subset feedback vertex set showing the claimed result.

Regarding the dependence of the exponent in the running time of the algorithm given in Theorem 8, note that we can hardly avoid this fact, since FEEDBACK VERTEx SET is $\mathrm{W}$ [1]-hard parameterized by the independent set number as explicitly given in [24]. At the same time such an observation follows from the W[1]-hardness result from the construction given in [22] with respect to the maximum induced matching width. In the full version of this extended abstract [32], we provide a different and simpler reduction from the MuLTiCOLORED INDEPENDENT SET problem [14,33] which shows an interesting connection with graphs of bounded independent set size.

\section{Extending to other Terminal Set Problems}

Let us now consider further terminal set problems that are related to SuBSET FEEDBACK Vertex Set. In these type of problems we are given a graph $G=(V, E)$, a terminal set $T \subseteq V$, and a nonnegative integer $k$ and the goal is to find a set $X \subseteq V$ with $|X| \leq k$ which intersects all "structures" (such as cycles or paths) passing through the vertices in $T$ [6]. In this setting, Subset FeEdBACK VERTEx SET is a particular terminal set problem when the objective structure is a cycle. We show that the ideas that we developed for SuBSET FeEdBack VerTex SeT on graphs of bounded independent set size, can be extended to further terminal set problems when the objective structure is a path instead of a cycle.

The (unweighted) Node Multiway Cut problem is concerned with finding a set $X \subseteq V \backslash T$ of size at most $k$ such that any path between two different terminals intersects $X$. Notice that in this problem we are not allowed to remove any terminal. For graphs having bounded independent set size, we completely characterize the complexity of NoDE Multiway Cut. In particular, for $\alpha(G)=3$ we can adopt the reduction given in Theorem 7 .

- Theorem 9. Let $G$ be a graph of independent set of size at most $d$. If $d \leq 2$ then Node Multiway Cut can be solved on time $n^{\mathcal{O}(1)}$. Otherwise, Node Multiway Cut is NP-complete on graphs of independent set of size at most 3. 
Due to the difficulty of Node Multiway Cut even for the unweighted version and with small independent set number, we consider a relaxed variation in which we are allowed to remove terminal vertices. The Node Multiway Cut with Deletable Terminals problem seeks for a solution $X$ with $X \subseteq V$ (instead of $X \subseteq V \backslash T)$. Next we show that the (unweighted) Node Multiway Cut with Deletable Terminals problem can be solved in polynomial time on graphs of bounded independent set number, using an idea similar to the one given in Theorem 8 .

- Theorem 10. Node Multiway Cut with Deletable Terminals on graphs of independent set of size at most $d$ can be solved in time $n^{\mathcal{O}(d)}$.

Proof. Let $(G, T, k)$ be an instance of Node Multiway Cut with Deletable Terminals where $G$ is a graph having independent set size at most $d$. Observe that every solution $X$ has size at most $|T|$. Assume first that $|T| \leq d$. Then we can enumerate all subsets having at most $|T|$ vertices in time $n^{\mathcal{O}(|T|)}$ and pick the smallest subset that separates all terminals. Thus in time $n^{\mathcal{O}(d)}$ we output a valid solution $X$, if it exists.

Next assume that $d<|T|$. We consider the graph $G[T]$. As an induced subgraph of $G$, $G[T]$ has independent set size at most $d$. Thus, $G[T]$ contains at least one edge. If both endpoints of an edge in $G[T]$ do not belong to solution $X$, then there is a path between terminal vertices. This means that there is a vertex cover $U$ of $G[T]$ such that $U \subseteq X$. To compute such a set $U$, we enumerate all independent sets $T^{\prime} \subseteq T$ of size at most $d$ in time $|T|^{\mathcal{O}(d)}$ and construct $U=T \backslash T^{\prime}$. For each constructed $U$, we consider the graph $G^{\prime}=G-U$ with terminals $T^{\prime}$. Since $T^{\prime}$ is an independent set in $G^{\prime}$, we know that $\left|T^{\prime}\right| \leq d$. Thus in time $n^{\mathcal{O}\left(\left|T^{\prime}\right|\right)}$ we can compute a set $X^{\prime}$ of minimum size such that all terminals of $G^{\prime}-X^{\prime}$ are separated. Therefore, the total running time is bounded by $|T|^{\mathcal{O}(d)} \cdot n^{\mathcal{O}\left(\left|T^{\prime}\right|\right)}$ which is bounded by $n^{\mathcal{O}(d)}$, because $|T| \leq n$ and $\left|T^{\prime}\right| \leq d$.

Let us also stress that we can hardly avoid the dependence of the exponent in the running time given in Theorem 10. This comes from the fact that Node Multiway Cut with Deletable Terminals with $T=V(G)$ is equivalent to asking whether the graph contains an independent set of size at least $k$. That is, we have to solve the InDEPENDENT SET which is known to be W[1]-hard parameterized by the size of the independent set [12].

Regarding the node-weighted variant of Node Multiway Cut with Deletable Terminals, we provide a dichotomy result with respect to $\alpha(G)$. In fact, for $\alpha(G) \leq 2$ we can invoke the algorithm for the Weighted Subset Feedback Vertex Set given in Theorem 6 , by adding a new vertex with a large weight that is adjacent to all terminals. Moreover, due to its close connection to the Node Multiway Cut, for $\alpha(G)>2$ we can assign appropriate weights to the terminals in a way that they become undeletable.

- Theorem 11. Let $G$ be a graph of independent set of size at most $d$. If $d \leq 2$ then Weighted Node Multiway Cut with Deletable Terminals can be solved in time $n^{\mathcal{O}(1)}$. Otherwise, Weighted Node Multiway Cut with Deletable Terminals is $N P$-complete on graphs of independent set of size at most 3 .

\section{Concluding Remarks}

Despite the fact that the Weighted Subset Feedback Vertex Set is NP-complete on graphs with bounded independent set number, it is still interesting to settle the complexity of Subset Feedback Vertex Set on graphs of maximum induced matching width by extending the approach given in [23]. Moreover, Feedback Vertex Set is known to be 
polynomially-time solvable on cocomparability graphs [28], and, more generally, on AT-free graphs [26]. To our knowledge, Subset Feedback Vertex Set has not been studied on such graphs, besides the existence of a fast exponential-time algorithm for the unweighted variant of the problem [6]. Furthermore, Theorem 9 shows that Node Multiway Cut remains NP-complete on graphs having maximum induced matching three. However, on graphs of bounded maximum induced matching the complexity of Node MultiwaY Cut with Deletable Terminals is still unknown.

\section{References}

1 A. Brandstädt, V. B. Le, and J. Spinrad. Graph Classes: A Survey. Society for Industrial and Applied Mathematics, 1999.

2 B.-M. Bui-Xuan, O. Suchý, J. A. Telle, and M. Vatshelle. Feedback vertex set on graphs of low clique-width. Eur. Journal of Combinatorics, 34(3):666-679, 2013.

3 G. Calinescu. Multiway Cut. In Encyclopedia of Algorithms. Springer, 2008.

4 J. Chen, Y. Liu, and S. Lu. An improved parameterized algorithm for the minimum node multiway cut problem. Algorithmica, 55:1-13, 2009.

5 R. H. Chitnis, F. V. Fomin, D. Lokshtanov, P. Misra, M. S. Ramanujan, and S. Saurabh. Faster exact algorithms for some terminal set problems. In Proceedings of IPEC 2013, pages 150-162, 2013.

6 R. H. Chitnis, F. V. Fomin, D. Lokshtanov, P. Misra, M. S. Ramanujan, and S. Saurabh. Faster exact algorithms for some terminal set problems. Journal of Computer and System Sciences, 88:195-207, 2017.

7 D. G. Corneil and J. Fonlupt. The complexity of generalized clique covering. Discrete Applied Mathematics, 22(2):109-118, 1988.

8 M. Cygan, J. Nederlof, M. Pilipczuk, M. Pilipczuk, J. M. M. van Rooij, and J. O. Wojtaszczyk. Solving connectivity problems parameterized by treewidth in single exponential time. In Proceedings of FOCS 2011, pages 150-159, 2011.

9 M. Cygan, M. Pilipczuk, M. Pilipczuk, and J. O. Wojtaszczyk. On multiway cut parameterized above lower bounds. ACM Trans. Comput. Theory, 5(1):3:1-3:11, 2013.

10 M. Cygan, M. Pilipczuk, M. Pilipczuk, and J. O. Wojtaszczyk. Subset feedback vertex set is fixed-parameter tractable. SIAM J. Discrete Math., 27(1):290-309, 2013.

11 R. Diestel. Graph Theory, 4th Edition, volume 173 of Graduate Texts in Mathematics. Springer, 2012.

12 R. G. Downey and M. R. Fellows. Fundamentals of Parameterized Complexity. Texts in Computer Science. Springer, 2013.

13 G. Even, J. Naor, and L. Zosin. An 8-approximation algorithm for the subset feedback vertex set problem. SIAM J. Comput., 30(4):1231-1252, 2000.

14 M. R. Fellows, D. Hermelin, F. A. Rosamond, and S. Vialette. On the parameterized complexity of multiple-interval graph problems. Theor. Comput. Sci., 410(1):53-61, 2009.

15 F. V. Fomin, S. Gaspers, D. Lokshtanov, and S. Saurabh. Exact algorithms via monotone local search. In Proceedings of STOC 2016, pages 764-775, 2016.

16 F. V. Fomin, P. Heggernes, D. Kratsch, C. Papadopoulos, and Y. Villanger. Enumerating minimal subset feedback vertex sets. Algorithmica, 69(1):216-231, 2014.

17 M. R. Garey and D. S. Johnson. Computers and Intractability. W. H. Freeman and Co., 1978.

18 N. Garg, V. V. Vazirani, and M. Yannakakis. Multiway cuts in node weighted graphs. J. Algorithms, 50(1):49-61, 2004.

19 P. A. Golovach, P. Heggernes, D. Kratsch, and R. Saei. Subset feedback vertex sets in chordal graphs. J. Discrete Algorithms, 26:7-15, 2014. 
20 M. C. Golumbic. Algorithmic Graph Theory and Perfect Graphs. Annals of Discrete Mathematics 57, Elsevier, 2004.

21 E. C. Hols and S. Kratsch. A randomized polynomial kernel for subset feedback vertex set. Theory Comput. Syst., 62:54-65, 2018.

22 L. Jaffke, O. Kwon, and J. A. Telle. A note on the complexity of feedback vertex set parameterized by mim-width. CoRR, abs/1711.05157, 2017.

23 L. Jaffke, O. Kwon, and J. A. Telle. A unified polynomial-time algorithm for feedback vertex set on graphs of bounded mim-width. In Proceedings of STACS 2018, pages 42:1-42:14, 2018 .

24 B. Jansen, V. Raman, and M. Vatshelle. Parameter ecology for feedback vertex set. Tsinghua Sci. and Technol., 19(4):387-409, 2014.

25 K. Kawarabayashi and Y. Kobayashi. Fixed-parameter tractability for the subset feedback set problem and the S-cycle packing problem. J. Comb. Theory, Ser. B, 102(4):1020-1034, 2012 .

26 D. Kratsch, H. Müller, and I. Todinca. Feedback vertex set on AT-free graphs. Discrete Applied Mathematics, 156(10):1936-1947, 2008.

27 S. Kratsch and M. Wahlstrom. Representative sets and irrelevant vertices: new tools for kernelization. In Proceedings of FOCS 2012, pages 450-459, 2012.

28 Y. D. Liang and M.-S. Chang. Minimum feedback vertex sets in cocomparability graphs and convex bipartite graphs. Acta Informatica, 34(5):337-346, 1997.

29 D. Marx. Parameterized graph separation problems. Theor. Comput. Sci., 351:399-406, 2006.

30 J. B. Orlin. Max flows in $\mathrm{O}(\mathrm{nm})$ time, or better. In Proceedings of STOC 2013, pages 765-774, 2013.

31 C. Papadopoulos and S. Tzimas. Polynomial-time algorithms for the subset feedback vertex set problem on interval graphs and permutation graphs. In Proceedings of FCT 2017, pages 381-394, 2017.

32 C. Papadopoulos and S. Tzimas. Subset feedback vertex set on graphs of bounded independent set size. CoRR, abs/1805.07141, 2018. arXiv: 1805.07141.

33 K. Pietrzak. On the parameterized complexity of the fixed alphabet shortest common supersequence and longest common subsequence problems. J. Comput. Syst. Sci., 67(4):757-771, 2003.

34 J. P. Spinrad. Efficient Graph Representations. American Mathematical Society, Fields Institute Monograph Series 19, 2003.

35 M. Vatshelle. New Width Parameters of Graphs. PhD thesis, University of Bergen, Norway, 2012.

36 M. Yannakakis. Node-deletion problems on bipartite graphs. SIAM J. Comput., 10(2):310327, 1981. 